\title{
Numerical approach of riemann-liouville fractional derivative operator
}

\author{
Ramzi B. Albadarneh ${ }^{1}$, Iqbal M. Batiha ${ }^{2}$, Ahmad Adwai ${ }^{3}$, Nedal Tahat ${ }^{4}$, A.K. Alomari ${ }^{5}$ \\ ${ }^{1,3,4}$ Department of Mathematics, The Hashemite University, Zarqa, Jordan \\ ${ }^{2}$ Department of Mathematics, Faculty of Science and Technology, Irbid National University, Irbid, Jordan \\ ${ }^{2}$ Nonlinear Dynamics Research Center (NDRC), Ajman University, Ajman, UAE \\ ${ }^{5}$ Department of Mathematics, Yarmouk University, Irbid, Jordan
}

\section{Article Info}

Article history:

Received Oct 30, 2020

Revised May 19, 2021

Accepted Jun 4, 2021

\section{Keywords:}

Fifth keyword

Fourth keyword

Fractional calculus

Riemann-liouville fractional

derivative operator

Weighted mean value theorem

\begin{abstract}
This article introduces some new straightforward and yet powerful formulas in the form of series solutions together with their residual errors for approximating the Riemann-Liouville fractional derivative operator. These formulas are derived by utilizing some of forthright computations, and by utilizing the so-called weighted mean value theorem (WMVT). Undoubtedly, such formulas will be extremely useful in establishing new approaches for several solutions of both linear and nonlinear fractionalorder differential equations. This assertion is confirmed by addressing several linear and nonlinear problems that illustrate the effectiveness and the practicability of the gained findings.
\end{abstract}

This is an open access article under the CC BY-SA license.

\section{Corresponding Author:}

Ramzi B. Albadarne

Department of Mathematics

The Hashemite University

Zarqa 13133, Jordan

Email: rbadarneh@hu.edu.jo

\section{INTRODUCTION}

The principle of fractional calculus has been endorsed as distinguished mathematical tools to characterize many real-world phenomena in the recent decades [1]-[5]. It has been increasingly considered by many researchers in numerous areas of engineering and science, some of these areas are and not limited to control engineering [6], electrochemistry [7], electromagnetism [8], bioscience [9], and diffusion processes [10]. Several different fractional derivatives and integrals definitions have been formulated and accepted, and they are divided into different categories. It's worth mentioning that there are two fractional derivative definitions; the first definition is the derivative of a function's convolution with a power law kernel, as suggested by Riemann and Liouville, the second is caputo's proposal of convolution of the local derivative of a given function with a power law function [11]. In view of different suggestions of many applied mathematicians, the caputo fractional derivative operator is acceptable for many real-world problems because it allows for the use of specified initial conditions when taking fractional derivatives, for instance, the Laplace transform [1], [12], [13]. Atangana et al. in [1] asserted that when a fractional integral operates, the initial function does not recover well, according to the mathematical definition of the caputo operator. As a result, although the caputo derivative is extremely useful and practical, it may not be appropriate for mathematical purposes [1]. The Riemann-Liouville operator, on the other hand, satisfies the mathematical principle in the fractional calculus sense. Furthermore, 
when using the Laplace transform, the initial condition with fractional exponent is taken into account, which is both practical and mathematically realistic [1].

In the same context, Taylor's series including fractional ones is suggested by many authors as one of the most efficient power series [14]. In 1847, the idea of the fractional generalized Taylor series was revealed, when Riemann used a series structure to formulate an analytic function [15]. The proof of the validity of such expansion for some classes of functions was given by Hardy [16]. Recently, the related mean value theorem problem was discussed by Trujillo et al. [17], and the result from the Riemann-Liouville case to the Caputo case was extended by Odibat et al. [18]. Another view to show fractional calculus, including Grünwald-Letnikov and Riemann-Liouville definitions has been suggested by Oldham and Spanier [19], also a power series involving integer derivatives of the analytic function was constructed [20]. Afterwards, to describe fractional derivative, a new series was proposed by Samko et al. [21]. In reference [22], the Taylor-Riemann series using Osler's theorem was investigated to obtain certain double infinite series expansions of some elementary functions. Some classical power series theorems have been generalized for fractional power series, and a new construction of the generalized Taylor's power series has been introduced in [14]. In reference [1], a numerical approximation of the Riemann-Liouville fractional derivative operator was presented. Analogues of the Taylor's theorem and the mean value theorem for fractional differential operators were established in reference [23]. More recently, Wei et al. have developed a general structure for Taylor series in fractional case by expanding an analytic function at the current time or at the initial instant [20]. This structure takes into account the Caputo definition, the Riemann-Liouville definition, the variable order and the constant order [20].

In this paper, a new straightforward formula in a series form for approximating the fractional derivative operator in the sense of Riemann-Liouville , $D^{\alpha} y(t), 0<\alpha \leq 1$, is introduced. Based on the weighted mean value theorem (WMVT) and some direct computations; this formula is derived. Because the solutions of some linear and nonlinear fractional differential equations are extremely difficult to obtain; such formula will be very useful to establish new approaches for them. These solutions will be in series forms that could be used in order to determine the analytic solutions in many cases. However the rest of this article is organized as follows: The Riemann-Liouville differential and integral operators are presented in section 2 with basic definitions and theorems. The theoretical framework is presented in section 3. Section 4 provides some examples to demonstrate the method. The final part of the paper is the conclusion.

\section{RIEMANN-LIOUVILLE DIFFERENTIAL AND INTEGRAL OPERATORS}

Calculus of integrals and derivatives of any arbitrary real or complex order is the topic of fractional calculus [24], [25]. As one of the most important fractional derivatives operators, the Riemann-Liouville operator satisfies all mathematical principles within the framework of fractional calculus [1], [26], [27]. To help researchers better understand how this operator generalizes ordinary differential operators, some definitions and properties related to this operator will be exhibited. Let us, firstly, assume that $[a, b]$ is a finite interval, where $a, b \in R$ and $-\infty<a<b<\infty$. The left-sided Riemann-Liouville fractional integral of order $\alpha \in R^{+}$is defined as [21], [28]:

$$
J_{a^{+}}^{\alpha} f(x)=\frac{1}{\Gamma(\alpha)} \int_{a}^{x} \frac{f(\tau)}{(x-\tau)^{1-\alpha}} d \tau, x>a,
$$

and the right-sided Riemann-Liouville fractional integral of order $\alpha \in R^{+}$is [28]:

$$
J_{b^{-}}^{\alpha} f(x)=\frac{1}{\Gamma(\alpha)} \int_{x}^{b} \frac{f(\tau)}{(\tau-x)^{1-\alpha}} d \tau, x<b .
$$

Observe that we have limited the values of the fractional order $\alpha$ to the real positive numbers [28], which is necessary for some practical applications, but one may find that $\alpha$ belongs to complex numbers in references [21], [28]. On the other hand, the left-sided Riemann-Liouville fractional derivative of order $\alpha \in R^{+}$is defined by [28]:

$$
D_{a^{+}}^{\alpha} f(x)=\frac{1}{\Gamma(n-\alpha)} \frac{d^{n}}{d x^{n}} \int_{a}^{x} \frac{f(\tau)}{(x-\tau)^{\alpha-n+1}} d \tau, x>a,
$$

and the right-sided Riemann-Liouville fractional derivative of order $\alpha \in R^{+}$is [28]: 


$$
D_{b^{-}}^{\alpha} f(x)=(-1)^{n} D^{n} J_{b^{-}}^{n-\alpha} f(x)=\frac{(-1)^{n}}{\Gamma(n-\alpha)} \frac{d^{n}}{d x^{n}} \int_{x}^{b} \frac{f(\tau)}{(\tau-x)^{\alpha-n+1}} d \tau, x<b,
$$

where $n=\lceil\alpha\rceil$, and where $\lceil\cdot\rceil$ denotes to the ceiling function. The popular forms of the Riemann-Liouville fractional integral and derivative of order $\alpha \in R^{+}$coincide with the left-sided Riemann-Liouville definitions. Actually, these forms can be defined as [29], [30], [31]:

$$
J_{a}^{\alpha} f(x)=\frac{1}{\Gamma(\alpha)} \int_{a}^{x} \frac{f(\tau)}{(x-\tau)^{1-\alpha}} d \tau, x>a,
$$

and

$$
D_{a}^{\alpha} f(x)=\frac{1}{\Gamma(n-\alpha)} \frac{d^{n}}{d x^{n}} \int_{a}^{x} \frac{f(\tau)}{(x-\tau)^{\alpha-n+1}} d \tau, x>a .
$$

It should be mentioned that the integral operators $J_{a^{+}}^{\alpha}, J_{b^{-}}^{\alpha}$ and $J_{a}^{\alpha}$ in (1), (2) and (5), respectively, are defined on $L^{p}(a, b) \equiv$ The space of integrable functions, where $p \in[1, \infty)$. At the same time, the differential operators $D_{a^{+}}^{\alpha}, D_{b^{-}}^{\alpha}$ and $D_{a}^{\alpha}$ in (3), (4) and (6), respectively, are defined on $C[a, b] \equiv$ The space of continuous functions [28]. Next, some important properties of the integral operator are stated for completeness.

Theorem 1: [32] Let $\alpha, \beta \geq 0$ and $\phi \in L^{1}[a, b]$. Then, $J_{a}^{\alpha} J_{a}^{\beta} \phi=J_{a}^{\alpha+\beta} \phi$ holds almost everywhere on $[a, b]$. If additionally $\phi \in C[a, b]$ or $\alpha+\beta \geq 1$, then the identity holds everywhere on $[a, b]$.

Corollary 2: [32] Let $\alpha, \beta \geq 0$ and $\phi \in L^{1}[a, b]$. Then, $J_{a}^{\alpha} J_{a}^{\beta} \phi=J_{a}^{\beta} J_{a}^{\alpha} \phi$.

Theorem 3: [30] The Riemann-Liouville fractional integral $J_{a}^{\alpha}$ of the power function satisfies:

$$
J_{a}^{\alpha}(x-a)^{\mu}=\frac{\Gamma(\mu+1)}{\Gamma(\mu+\alpha+1)}(x-a)^{\mu+\alpha}, \alpha>0, \mu>-1 .
$$

Having stated some fundamental properties of the Riemann-Liouville integral operator, we are now ready to state some properties of the corresponding differential operator.

Theorem 4: [32] Let $\alpha \geq 0$. Then for every $f \in L^{1}[a, b]$, we have $D_{a}^{\alpha} J_{a}^{\alpha} f(x)=f(x)$ almost everywhere.

Theorem 5: [32] Let $\alpha>0$, If there exists some $\phi \in L^{1}[a, b]$ such that $f=J_{a}^{\alpha} \phi$, then, $J_{a}^{\alpha} D_{a}^{\alpha} f(x)=f(x)$ almost everywhere.

Theorem 6: [32] Let $\alpha>0$ and $n-1 \leq \alpha<n, n \in N$. Assume that $f$ is such that $J_{a}^{(n-\alpha)} f \in A^{n}[a, b] \equiv$ The set of all functions with an absolutely continuous $(n-1)^{\text {th }}$ derivative. Then,

$$
J_{a}^{\alpha} D_{a}^{\alpha} f(x)=f(x)-\sum_{k=0}^{n-1} \frac{(x-a)^{\alpha-k-1}}{\Gamma(\alpha-k)} \lim _{z \rightarrow a^{+}} D^{(n-k-1)} J_{a}^{(n-\alpha)} f(z) .
$$

In particular, for $0<\alpha<1$, we have:

$$
J_{a}^{\alpha} D_{a}^{\alpha} f(x)=f(x)-\frac{(x-a)^{\alpha-1}}{\Gamma(\alpha)} \lim _{z \rightarrow a^{+}} J_{a}^{(1-\alpha)} f(z) .
$$

Theorem 7: [32] The Riemann-Liouville fractional derivative $D_{a}^{\alpha}$ of the power function satisfies:

$$
D_{a}^{\alpha}(x-a)^{\mu}=\frac{\Gamma(\mu+1)}{\Gamma(\mu+1-\alpha)}(x-a)^{(\mu-\alpha)}, \text { if } \alpha-\mu \notin N
$$

\section{THE THEORETICAL FRAMEWORK}

This section illustrates the theoretical framework of the present study. Actually, it introduces two novel theorems that offer two powerful expressions formulated in the form of power series to approximate the Riemann-Liouville fractional derivative operator. In summary, here are the main results of this work.

Theorem 8: Let $y \in C^{n+1}[a, b], 0<\alpha \leq 1$, and $a \geq 0$. Then for every $t \in(a, b]$, there exist $\xi \in(a, b)$ such that the Riemann-Liouville fractional derivative operator $D_{a}^{\alpha} y(t)$ can be written, in terms of fractional series 
and its reminder term, in the following form:

$$
\begin{aligned}
& D_{a}^{\alpha} y(t) \\
& =\frac{1}{\Gamma(1-\alpha)}\left(y(a)(t-a)^{-\alpha}+\sum_{k=1}^{n} \frac{y^{(k)}(a)(t-a)^{k-\alpha}}{\prod_{j=1}^{k}(j-\alpha)}+\frac{y^{(n+1)}(\xi)(t-a)^{n+1-\alpha}}{\prod_{j=1}^{n+1}(j-\alpha)}\right) .
\end{aligned}
$$

The Riemann-Liouville fractional derivative operator for $0<\alpha \leq 1$ is known as:

$$
D_{a}^{\alpha} y(t)=\frac{1}{\Gamma(1-\alpha)} \frac{d}{d t} \int_{a}^{t} y(x)(t-x)^{-\alpha} d x .
$$

Using integration by part to (10) yields:

$$
D_{a}^{\alpha} y(t)=\frac{1}{\Gamma(1-\alpha)}\left(y(a)(t-a)^{-\alpha}+\int_{a}^{t} y^{\prime}(x)(t-x)^{-\alpha} d x\right) .
$$

Again, applying integration by part inductively $n$-times to (11) leads to the following assertion:

$$
\begin{aligned}
& D_{a}^{\alpha} y(t)=\frac{1}{\Gamma(1-\alpha)}\left(y(a)(t-a)^{-\alpha}+\frac{y^{\prime}(a)(t-a)^{1-\alpha}}{1-\alpha}+\frac{y^{\prime \prime}(a)(t-a)^{2-\alpha}}{(1-\alpha)(2-\alpha)}\right)+ \\
& \cdots+\frac{1}{\Gamma(1-\alpha)}\left(\frac{y^{(n)}(a)(t-a)^{n-\alpha}}{\prod_{k=1}^{n}(k-\alpha)}+\frac{1}{\prod_{k=1}^{n}(k-\alpha)} \int_{a}^{t} \frac{y^{(n+1)}(x)(t-x)^{1-\alpha}}{1-\alpha} d x\right) .
\end{aligned}
$$

Observe that $y \in C^{n+1}[a, b]$ and $(t-x)^{n-\alpha}$ does not change its sign in $[a, t]$. Therefore, one can conclude using the WMVT that there exist $\xi \in(a, b)$ such that:

$$
\begin{aligned}
\int_{a}^{t} \frac{y^{(n+1)}(x)(t-x)^{n-\alpha}}{\prod_{k=1}^{n}(k-\alpha)} d x & =\frac{y^{(n+1)}(\xi)}{\prod_{k=1}^{n}(k-\alpha)} \int_{a}^{t}(t-x)^{n-\alpha} d x \\
& =\frac{y^{(n+1)}(\xi)(t-a)^{n+1-\alpha}}{(n+1-\alpha) \prod_{k=1}^{n}(k-\alpha)} \\
& =\frac{y^{(n+1)}(\xi)(t-a)^{n+1-\alpha}}{\prod_{k=1}^{n+1}(k-\alpha)},
\end{aligned}
$$

which consequently implies the desired result.

Theorem 9: Let $y \in C^{n+m}[a, b], a \geq 0$, and $m-1<\alpha<m$, where $m$ is positive integer. Then for every $t \in(a, b]$, there exist $\xi \in(a, b)$ such that the Riemann-Liouville fractional derivative operator $D_{a}^{\alpha} y(t)$ can be written, in terms of fractional series and its reminder term, in the following form:

$$
\begin{aligned}
& D_{a}^{\alpha} y(t) \\
& =\frac{1}{\Gamma(1-\alpha)}\left(y(a)(t-a)^{-\alpha}+\sum_{k=1}^{n} \frac{y^{(k)}(a)(t-a)^{k-\alpha}}{\prod_{j=1}^{k}(j-\alpha)}+\frac{y^{(n+1)}(\xi)(t-a)^{n+1-\alpha}}{\prod_{j=1}^{n+1}(j-\alpha)}\right) .
\end{aligned}
$$

The Riemann-Liouville operator given in (6) can be rewritten in the following form:

$$
\begin{aligned}
D_{a}^{\alpha} y(t)= & \frac{1}{(m-\alpha) \Gamma(m-\alpha)}\left(y(a)(m-\alpha)(m-\alpha-1) \ldots(1-\alpha)(t-a)^{-\alpha}\right. \\
& \left.+\frac{d^{m}}{d t^{m}}\left(\int_{a}^{t} y^{\prime}(x)(t-x)^{m-\alpha} d x\right)\right) .
\end{aligned}
$$

But, based on the following assertion:

$$
\frac{d^{m}}{d t^{m}}\left(\int_{a}^{t} y^{\prime}(x)(t-x)^{m-\alpha} d x\right)=\int_{a}^{t} y^{\prime}(x)(m-\alpha)(m-\alpha-1) \ldots(1-\alpha)(t-x)^{1-\alpha} d x
$$


in (15) will be as (16):

$$
D_{a}^{\alpha} y(t)=\frac{1}{\Gamma(1-\alpha)}\left(y(a)(t-a)^{-\alpha}+\int_{a}^{t} y^{\prime}(x)(t-x)^{-\alpha} d x\right) .
$$

Finally, using the same proof of Theorem 8 yields also the desired result.

\section{ILLUSTRATIVE NUMERICAL EXAMPLES}

In this part, the effectiveness and efficiency of our findings are numerically verified through solving some linear and nonlinear fractional differential equations.

Example 10: Consider the function $y(t)=-\exp (-t)+t^{8}+\cos \left(\frac{\pi t^{2}}{3}\right)-6 t$. The two formulas (9) and (10) given, respectively, in theorem 8 and theorem 9 are employed via mathematica package in order to gain the approximate values of the Riemann-Liouville fractional derivative operator $D_{a}^{\alpha} y(t)$ versus the time $t$ for different values of $\alpha$ and $n$. However, Tables 1,2,3, and 4 show the error terms between the approximate and the exact values. One can observe that these errors can be reduced by sufficiently increasing the value of $n$ or by reducing the round of error that could be occurred when applying mathematica package.

Table 1. Error terms for example 10

\begin{tabular}{ccccc}
\hline $\mathrm{t}$ & $\alpha=0.5, \mathrm{n}=20$ & $\alpha=0.7, \mathrm{n}=20$ & $\alpha=0.9, \mathrm{n}=20$ & $\alpha=0.99, \mathrm{n}=20$ \\
\hline 0.1 & 0.00 & $8.88 \times 10^{-16}$ & 0.00 & $8.88 \times 10^{-16}$ \\
0.2 & 0.00 & $1.78 \times 10^{-15}$ & $1.78 \times 10^{-15}$ & $8.88 \times 10^{-16}$ \\
0.3 & $8.88 \times 10^{-16}$ & $1.78 \times 10^{-15}$ & $8.88 \times 10^{-16}$ & $1.78 \times 10^{-15}$ \\
0.4 & $8.88 \times 10^{-16}$ & $1.78 \times 10^{-15}$ & 0.00 & $8.88 \times 10^{-16}$ \\
0.5 & $1.78 \times 10^{-15}$ & $2.66 \times 10^{-15}$ & $4.44 \times 10^{-15}$ & $1.07 \times 10^{-14}$ \\
0.6 & $1.09 \times 10^{-13}$ & $2.26 \times 10^{-13}$ & $4.75 \times 10^{-13}$ & $6.62 \times 10^{-13}$ \\
0.7 & $4.09 \times 10^{-12}$ & $8.28 \times 10^{-12}$ & $1.68 \times 10^{-11}$ & $2.30 \times 10^{-11}$ \\
0.8 & $9.42 \times 10^{-11}$ & $1.86 \times 10^{-10}$ & $3.66 \times 10^{-10}$ & $4.96 \times 10^{-10}$ \\
0.9 & $1.50 \times 10^{-9}$ & $2.88 \times 10^{-9}$ & $5.55 \times 10^{-9}$ & $7.44 \times 10^{-9}$ \\
1.0 & $1.78 \times 10^{-8}$ & $3.35 \times 10^{-8}$ & $6.31 \times 10^{-8}$ & $8.38 \times 10^{-8}$ \\
\hline
\end{tabular}

Table 2. Error terms for example 10

\begin{tabular}{ccccc}
\hline $\mathrm{t}$ & $\alpha=0.5, \mathrm{n}=50$ & $\alpha=0.7, \mathrm{n}=50$ & $\alpha=0.9, \mathrm{n}=50$ & $\alpha=0.99, \mathrm{n}=50$ \\
\hline 0.1 & 0.00 & $1.33 \times 10^{-15}$ & 0.00 & 0.00 \\
0.2 & 0.00 & $2.22 \times 10^{-15}$ & $1.78 \times 10^{-15}$ & 0.00 \\
0.3 & 0.00 & $1.78 \times 10^{-15}$ & 0.00 & $1.78 \times 10^{-15}$ \\
0.4 & $8.88 \times 10^{-16}$ & $1.78 \times 10^{-15}$ & 0.00 & $1.78 \times 10^{-15}$ \\
0.5 & 0.00 & 0.00 & $1.78 \times 10^{-15}$ & $8.88 \times 10^{-16}$ \\
0.6 & 0.00 & $3.55 \times 10^{-15}$ & $1.78 \times 10^{-15}$ & $8.88 \times 10^{-16}$ \\
0.7 & 0.00 & $3.55 \times 10^{-15}$ & $8.88 \times 10^{-16}$ & $1.78 \times 10^{-15}$ \\
0.8 & $8.88 \times 10^{-16}$ & $3.55 \times 10^{-15}$ & $1.78 \times 10^{-15}$ & 0.00 \\
0.9 & $1.78 \times 10^{-15}$ & $6.22 \times 10^{-15}$ & $8.88 \times 10^{-16}$ & $1.33 \times 10^{-15}$ \\
1.0 & $8.88 \times 10^{-16}$ & $1.02 \times 10^{-14}$ & $1.89 \times 10^{-15}$ & $4.44 \times 10^{-16}$ \\
\hline
\end{tabular}

Example 11: Consider the following nonlinear fractional IVP:

$$
D_{0}^{\alpha} y(t)=t^{1-\alpha}(1-y)^{2}, \quad y(0)=0 .
$$

The exact solution of this problem is $y(t)=\frac{t}{1+t}$, for $\alpha=1$. However, in order to employ our proposed scheme to solve such problem, one can firstly take the Taylor's series around $t=0$ for the left-hand side of (17), and then use the result reported in theorem $8 \mathrm{In}$ other words, $D_{0}^{\alpha} y(t)$ can be replaced by the following assertion:

$$
D_{0}^{\alpha} y(t)=\frac{1}{\Gamma(1-\alpha)}\left(y(0) t^{-\alpha}+\sum_{k=1}^{\infty} \frac{y^{(k)}(0) t^{k-\alpha}}{\prod_{j=1}^{k}(j-\alpha)}\right) .
$$


Using the initial condition $y(0)=0$ implies:

$$
\begin{aligned}
\frac{t^{1-\alpha} y^{\prime}(0)}{\Gamma(2-\alpha)}+\frac{t^{2-\alpha} y^{\prime \prime}(0)}{\Gamma(3-\alpha)}+\frac{y^{(3)}(0) t^{3-\alpha}}{\Gamma(4-\alpha)}+\ldots \\
\quad=t^{1-\alpha}-2 t^{2-\alpha} y^{\prime}(0)+t^{3-\alpha}\left(y^{\prime}(0)^{2}-y^{\prime \prime}(0)\right)+\ldots
\end{aligned}
$$

Equating the coefficients of $t^{j}$ of (19) yields:

$$
\begin{aligned}
y^{\prime}(0) & =\Gamma(2-\alpha), \\
y^{\prime \prime}(0) & =-2 \Gamma(2-\alpha) \Gamma(3-\alpha), \\
y^{\prime \prime \prime}(0) & =\left(\Gamma(2-\alpha)^{2}+2 \Gamma(3-\alpha) \Gamma(2-\alpha)\right) \Gamma(4-\alpha),
\end{aligned}
$$

consequently, substituting each of $y(0), y^{\prime}(0), y^{\prime \prime}(0), \cdots$ into the power series of $y(t)$ around $t=0$ leads us to establish the general solution of (17), which would be in the following form:

$$
\begin{aligned}
& y(t)=t \Gamma(2-\alpha)-t^{2} \Gamma(2-\alpha) \Gamma(3-\alpha)+\frac{1}{6} t^{3}\left[\Gamma(2-\alpha)^{2}+2 \Gamma(3-\alpha) \Gamma(2-\alpha)\right] \\
& \times \Gamma(4-\alpha)+\frac{1}{72} t^{4}\left[-6 \Gamma(3-\alpha) \Gamma(2-\alpha)^{2}-\left[\Gamma(2-\alpha)^{2}+2 \Gamma(3-\alpha) \Gamma(2-\alpha)\right]\right. \\
& \times \Gamma(4-\alpha)] \Gamma(5-\alpha)+\ldots
\end{aligned}
$$

One can easily verify that, when $\alpha=1$, this solution would be the same exact solution given above, i.e;

$$
y(t)=t-t^{2}+t^{3}-t^{4}+t^{5}-t^{6}+t^{7}-t^{8}+t^{9}-t^{10}+\ldots=\frac{t}{1+t} .
$$

In particular, the power series solution of the IVP given in (17), for $0<\alpha \leq 1$, can be written as:

$$
y(t)=\sum_{m=0}^{\infty} \frac{c(m) t^{m}}{m !}
$$

where

$$
c(0)=0, c(1)=\Gamma(2-\alpha)
$$

and

$$
c(m)=\Gamma(m-\alpha+1)\left(\sum_{k=0}^{m-1} \frac{c(k) c(-k+m-1)}{k !(-k+m-1) !}-\frac{2 c(m-1)}{(m-1) !}\right) .
$$

Table 3. Error terms for example 10

\begin{tabular}{ccccc}
\hline $\mathrm{t}$ & $\alpha=1.5, \mathrm{n}=20$ & $\alpha=1.7, \mathrm{n}=20$ & $\alpha=1.9, \mathrm{n}=20$ & $\alpha=1.99, \mathrm{n}=20$ \\
\hline 0.1 & 0.00 & $2.84 \times 10^{-14}$ & $9.95 \times 10^{-14}$ & $2.66 \times 10^{-14}$ \\
0.2 & 0.00 & $7.11 \times 10^{-15}$ & $2.13 \times 10^{-14}$ & $7.99 \times 10^{-15}$ \\
0.3 & $3.55 \times 10^{-15}$ & $3.55 \times 10^{-15}$ & $1.42 \times 10^{-14}$ & $5.33 \times 10^{-15}$ \\
0.4 & 0.00 & 0.00 & $1.07 \times 10^{-14}$ & $1.33 \times 10^{-15}$ \\
0.5 & $6.93 \times 10^{-14}$ & $1.51 \times 10^{-13}$ & $3.33 \times 10^{-13}$ & $4.57 \times 10^{-13}$ \\
0.6 & $4.28 \times 10^{-12}$ & $8.87 \times 10^{-12}$ & $1.83 \times 10^{-11}$ & $2.54 \times 10^{-11}$ \\
0.7 & $1.37 \times 10^{-10}$ & $2.76 \times 10^{-10}$ & $5.53 \times 10^{-10}$ & $7.55 \times 10^{-10}$ \\
0.8 & $2.76 \times 10^{-9}$ & $5.41 \times 10^{-9}$ & $1.06 \times 10^{-8}$ & $1.43 \times 10^{-8}$ \\
0.9 & $3.91 \times 10^{-8}$ & $7.46 \times 10^{-8}$ & $1.42 \times 10^{-7}$ & $1.90 \times 10^{-7}$ \\
1.0 & $4.17 \times 10^{-7}$ & $7.80 \times 10^{-7}$ & $1.46 \times 10^{-6}$ & $1.93 \times 10^{-6}$ \\
\hline
\end{tabular}


Table 4. Error terms for example 10

\begin{tabular}{ccccc}
\hline $\mathrm{t}$ & $\alpha=1.5, \mathrm{n}=50$ & $\alpha=1.7, \mathrm{n}=50$ & $\alpha=1.9, \mathrm{n}=50$ & $\alpha=1.99, \mathrm{n}=50$ \\
\hline 0.1 & 0.00 & 0.00 & $9.95 \times 10^{-14}$ & $2.66 \times 10^{-14}$ \\
0.2 & $1.42 \times 10^{-14}$ & $7.11 \times 10^{-15}$ & $2.13 \times 10^{-14}$ & $7.99 \times 10^{-15}$ \\
0.3 & $3.55 \times 10^{-15}$ & $3.55 \times 10^{-15}$ & $1.42 \times 10^{-14}$ & $4.88 \times 10^{-15}$ \\
0.4 & 0.00 & 0.00 & $8.88 \times 10^{-15}$ & $4.88 \times 10^{-15}$ \\
0.5 & $3.55 \times 10^{-15}$ & $1.78 \times 10^{-15}$ & $7.11 \times 10^{-15}$ & $3.55 \times 10^{-15}$ \\
0.6 & 0.00 & 0.00 & $4.88 \times 10^{-15}$ & $2.22 \times 10^{-16}$ \\
0.7 & $1.78 \times 10^{-15}$ & $6.22 \times 10^{-15}$ & $3.16 \times 10^{-15}$ & $7.11 \times 10^{-15}$ \\
0.8 & $8.88 \times 10^{-16}$ & $1.62 \times 10^{-14}$ & $8.88 \times 10^{-16}$ & $2.66 \times 10^{-14}$ \\
0.9 & $4.22 \times 10^{-15}$ & $2.13 \times 10^{-14}$ & $3.55 \times 10^{-15}$ & $7.46 \times 10^{-14}$ \\
1.0 & $1.78 \times 10^{-15}$ & $6.04 \times 10^{-14}$ & $7.11 \times 10^{-15}$ & $1.21 \times 10^{-13}$ \\
\hline
\end{tabular}

Example 12: Consider the following nonlinear fractional IVP that describes the cooling of a semi-infinite body by radiation:

$$
D_{a}^{\alpha} y(t)=t^{1-\alpha}(1-y(t))^{4}, \quad y(0)=0 .
$$

The exact solution of (20) for $\alpha=1$ is of the form:

$$
y(t)=1-\frac{\left(1+6 t+9 t^{2}\right)^{\frac{1}{3}}}{(1+3 t)} .
$$

Following the same technique applied to example 11 leads us to deduce the general solution of (20). This solution can be written in the following form:

$$
\begin{aligned}
& y(t)=t \Gamma(2-\alpha)-2 t^{2} \Gamma(2-\alpha) \Gamma(3-\alpha)+\frac{1}{3} t^{3}\left[3 \Gamma(2-\alpha)^{2}+4 \Gamma(3-\alpha) \Gamma(2-\alpha)\right] \\
& \times \Gamma(4-\alpha)+\frac{1}{24} t^{4}\left[-4 \Gamma(2-\alpha)^{3}-24 \Gamma(3-\alpha) \Gamma(2-\alpha)^{2}-\frac{4}{3}\left[3 \Gamma(2-\alpha)^{2}\right.\right. \\
& +4 \Gamma(3-\alpha) \Gamma(2-\alpha)] \Gamma(4-\alpha)] \Gamma(5-\alpha)+\ldots
\end{aligned}
$$

For $\alpha=1$, the power series solution will be as follows:

$$
y(t)=t-2 t^{2}+\frac{14 t^{3}}{3}-\frac{35 t^{4}}{3}+\frac{91 t^{5}}{3}+\ldots,
$$

which coincides exactly with the power series of (21). However, Table 5 shows the approximate solutions together with their residual errors of (21) for different values of $n$ and $\alpha$. Besides, Figure 1 shows the exact and the approximate solution of (21) for different values of $\alpha$ at $n=20$. In view of these numerical results, it can be asserted that the residual error is decreased when $n$ becomes large.

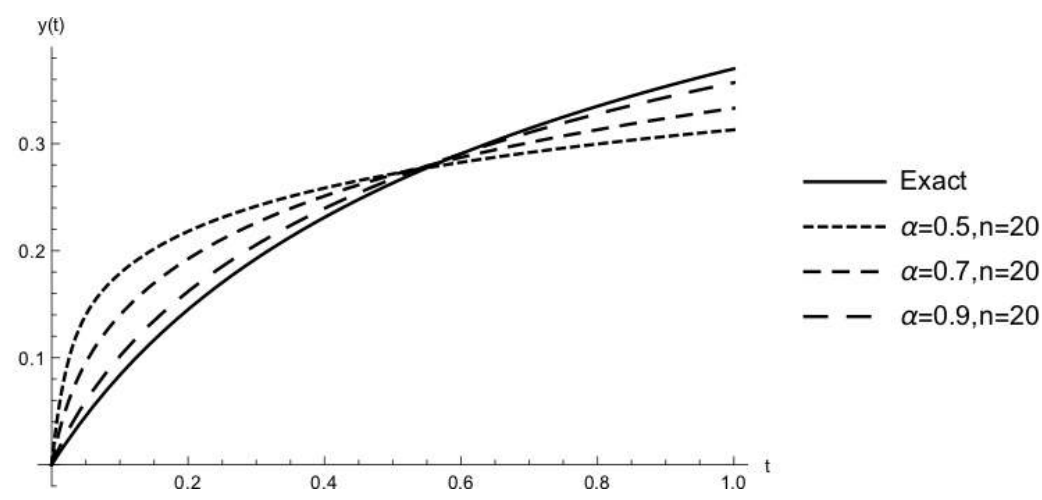

Figure 1. An approximate solution for example 12 for different values of $\alpha$ 
Table 5. Numerical solutions of example 12 together with their residual errors for different values of $t$

\begin{tabular}{|c|c|c|c|c|c|c|c|c|}
\hline \multirow[b]{2}{*}{ (r)2-3(r)4-5(r)6-7(r)4-5(r)8-9t } & \multicolumn{2}{|c|}{$\alpha=0.5, n=10$} & \multicolumn{2}{|c|}{$\alpha=0.5, n=20$} & \multicolumn{2}{|c|}{$\alpha=0.7, n=10$} & \multicolumn{2}{|c|}{$\alpha=0.7, n=20$} \\
\hline & app. & Res. & app. & Res. & app. & Res. & app. & Res. \\
\hline 0.1 & 0.1693669 & $\begin{array}{c}1.74 \times \\
10^{-6}\end{array}$ & 0.1787661 & $\begin{array}{l}4.44 \times \\
10^{-11}\end{array}$ & 0.1326126 & $\begin{array}{c}5.31 \times \\
10^{-7}\end{array}$ & 0.1398065 & $\begin{array}{l}2.76 \times \\
10^{-11}\end{array}$ \\
\hline 0.2 & 0.2144900 & $\begin{array}{c}1.17 \times \\
10^{-5}\end{array}$ & 0.2180800 & $\begin{array}{c}1.44 \times \\
10^{-9}\end{array}$ & 0.1888217 & $\begin{array}{c}4.05 \times \\
10^{-6}\end{array}$ & 0.1923520 & $\begin{array}{l}8.08 \times \\
10^{-12}\end{array}$ \\
\hline 0.3 & 0.2391206 & $\begin{array}{c}4.15 \\
10^{-5}\end{array}$ & 0.2416223 & $\begin{array}{c}1.36 \times \\
10^{-8}\end{array}$ & 0.2236491 & $\begin{array}{c}1.56 \times \\
10^{-5}\end{array}$ & 0.2261308 & $\begin{array}{c}3.01 \times \\
10^{-9}\end{array}$ \\
\hline 0.4 & 0.2567284 & $\begin{array}{c}9.36 \times \\
10^{-5}\end{array}$ & 0.2585229 & $\begin{array}{c}5.23 \times \\
10^{-8}\end{array}$ & 0.2492026 & $\begin{array}{c}3.71 \times \\
10^{-5}\end{array}$ & 0.2510679 & $\begin{array}{c}1.38 \times \\
10^{-8}\end{array}$ \\
\hline 0.5 & 0.2702355 & $\begin{array}{c}1.44 \times \\
10^{-4}\end{array}$ & 0.2717184 & $\begin{array}{c}9.45 \times \\
10^{-8}\end{array}$ & 0.2692866 & $\begin{array}{c}5.97 \times \\
10^{-5}\end{array}$ & 0.2708023 & $\begin{array}{c}2.72 \times \\
10^{-8}\end{array}$ \\
\hline 0.6 & 0.2813543 & $\begin{array}{c}1.55 \times \\
10^{-4}\end{array}$ & 0.2825414 & $\begin{array}{c}8.41 \times \\
10^{-8}\end{array}$ & 0.2858447 & $\begin{array}{c}6.68 \times \\
10^{-5}\end{array}$ & 0.2871004 & $\begin{array}{c}2.64 \times \\
10^{-8}\end{array}$ \\
\hline 0.7 & 0.2906744 & $\begin{array}{c}1.18 \times \\
10^{-4}\end{array}$ & 0.2917126 & $\begin{array}{c}3.32 \times \\
10^{-8}\end{array}$ & 0.2998796 & $\begin{array}{c}5.25 \times \\
10^{-5}\end{array}$ & 0.3009584 & $\begin{array}{c}9.88 \times \\
10^{-9}\end{array}$ \\
\hline 0.8 & 0.2987601 & $\begin{array}{c}6.25 \times \\
10^{-5}\end{array}$ & 0.2996669 & $\begin{array}{c}8.93 \times \\
10^{-9}\end{array}$ & 0.3120482 & $\begin{array}{c}2.85 \times \\
10^{-5}\end{array}$ & 0.3129942 & $\begin{array}{c}2.50 \times \\
10^{-9}\end{array}$ \\
\hline 0.9 & 0.3059084 & $\begin{array}{c}2.19 \times \\
10^{-5}\end{array}$ & 0.3066869 & $\begin{array}{c}2.87 \times \\
10^{-8}\end{array}$ & 0.3227899 & $\begin{array}{c}1.02 \times \\
10^{-5}\end{array}$ & 0.3236183 & $\begin{array}{c}2.80 \times \\
10^{-9}\end{array}$ \\
\hline 1.0 & 0.3121975 & $\begin{array}{c}4.58 \times \\
10^{-6}\end{array}$ & 0.3129633 & $\begin{array}{c}3.13 \times \\
10^{-8}\end{array}$ & 0.3323502 & $\begin{array}{c}2.19 \times \\
10^{-6}\end{array}$ & 0.3331162 & $\begin{array}{c}4.32 \times \\
10^{-9}\end{array}$ \\
\hline
\end{tabular}

Example 13: Consider the following nonlinear fractional IVP; Riccati differential equation:

$$
D_{a}^{\alpha} y(t)=t^{1-\alpha}+2 t^{1-\alpha} y(t)-t^{1-\alpha} y(t)^{2}, \quad y(0)=0 .
$$

The exact solution for $\alpha=1$ is of the form:

$$
y(t)=\frac{e^{2 \sqrt{2} t}-1}{\sqrt{2} e^{2 \sqrt{2} t}-e^{2 \sqrt{2} t}+\sqrt{2}+1} .
$$

Likewise example 11, we found the general solution of (22) as:

$$
\begin{aligned}
& y(t)=\left[t+t^{2} \Gamma(3-\alpha)\right] \Gamma(2-\alpha)-\frac{t^{3} \Gamma(4-\alpha)}{6}\left[\Gamma(2-\alpha)^{2}-2 \Gamma(2-\alpha) \Gamma(3-\alpha)\right] \\
& -\frac{1}{72} t^{4}\left[6 \Gamma(3-\alpha) \Gamma(2-\alpha)^{2}+\left[\Gamma(2-\alpha)^{2}-2 \Gamma(2-\alpha) \Gamma(3-\alpha)\right] \Gamma(4-\alpha)\right] \Gamma(5-\alpha) \\
& +\cdots
\end{aligned}
$$

For $\alpha=1$, the power series solution is of the form:

$$
y(t)=t+t^{2}+\frac{t^{3}}{3}-\frac{t^{4}}{3}-\frac{7 t^{5}}{15}+\cdots,
$$

which coincides exactly with the power series of (23). However, Table 6 shows the approximate solution together with its residual error of (23) for different values of $n$ and $\alpha$. Besides, Figure 2 shows the exact and the approximate solution of (22) for different values of $\alpha$ at $n=20$.

Example 14: Consider the following nonlinear fractional IVP:

$$
D_{a}^{\alpha} y(t)=t^{2-\alpha}\left(3-y^{\prime}(t)\right)^{2 / 3}, \quad y(0)=0, y^{\prime}(0)=0 .
$$

The exact solution of (24) for $\alpha=2$ is of the form:

$$
y(t)=\frac{1}{108} t^{2}\left(t^{2}-12 \sqrt[3]{3} t+543^{2 / 3}\right)
$$


The same technique applied to example 11 can be also applied here to find the general solution of (24) for $1<\alpha<2$. This solution is, however, of the form:

$$
\begin{aligned}
y(t) & =\frac{1}{2} 3^{2 / 3} t^{2} \Gamma(3-\alpha)-\frac{t^{3} \Gamma(3-\alpha) \Gamma(4-\alpha)}{33^{2 / 3}}+\frac{1}{24} t^{4}\left[\frac{2}{9} \Gamma(3-\alpha) \Gamma(4-\alpha)\right. \\
& \left.-\frac{\Gamma(3-\alpha)^{2}}{9}\right] \Gamma(5-\alpha)+\frac{1}{120} t^{5}\left[-\frac{4 \Gamma(3-\alpha)^{3}}{81 \sqrt[3]{3}}+\frac{2 \Gamma(4-\alpha)^{3}}{27 \sqrt[3]{3}}\right. \\
& \left.-\frac{\Gamma(5-\alpha)\left[\frac{2}{9} \Gamma(3-\alpha) \Gamma(4-\alpha)-\frac{\Gamma(3-\alpha)^{2}}{9}\right]}{9 \sqrt[3]{3}}\right] \Gamma(6-\alpha)+\cdots
\end{aligned}
$$

Observe that the power series solution, for $\alpha=2$, is of the form:

$$
y(t)=\frac{1}{2} 3^{2 / 3} t^{2}-\frac{t^{3}}{33^{2 / 3}} \frac{t^{4}}{108}+\cdots
$$

This solution is exactly equals the power series of (25). For more insight, Table 7 shows the approx-

\begin{tabular}{|c|c|c|c|c|c|c|c|c|}
\hline (r)2-3(r)4-5(r)6-7(r)4-5(r)8-9 & \multicolumn{2}{|c|}{$\alpha=0.5, n=10$} & \multicolumn{2}{|c|}{$\alpha=0.5, n=20$} & \multicolumn{2}{|c|}{$\alpha=0.7, n=10$} & \multicolumn{2}{|c|}{$\alpha=0.7, n=20$} \\
\hline 0.1 & 0.540394230 & $\begin{array}{c}9.72 \times \\
10^{-7}\end{array}$ & 0.569770720 & $\begin{array}{l}2.73 \times \\
10^{-10}\end{array}$ & 0.269035900 & $\begin{array}{c}1.07 \times \\
10^{-7}\end{array}$ & 0.281235570 & $\begin{array}{l}3.98 \times \\
10^{-12}\end{array}$ \\
\hline 0.2 & 0.897926500 & $\begin{array}{l}6.89 \times \\
10^{-12}\end{array}$ & 0.917109000 & $\begin{array}{c}2.44 \times \\
10^{-6}\end{array}$ & 0.525631260 & $\begin{array}{c}9.39 \times \\
10^{-9}\end{array}$ & 0.537244240 & $\begin{array}{l}1.39 \times \\
10^{-10}\end{array}$ \\
\hline 0.3 & 1.149028300 & $\begin{array}{c}2.34 \times \\
10^{-5}\end{array}$ & 1.162603400 & $\begin{array}{c}6.82 \times \\
10^{-9}\end{array}$ & 0.776353670 & $\begin{array}{c}3.87 \times \\
10^{-6}\end{array}$ & 0.787393800 & $\begin{array}{c}1.65 \times \\
10^{-9}\end{array}$ \\
\hline 0.5 & 1.460432400 & $\begin{array}{c}7.19 \times \\
10^{-5}\end{array}$ & 1.467716300 & $\begin{array}{c}5.41 \times \\
10^{-8}\end{array}$ & 1.213574200 & $\begin{array}{c}1.50 \times \\
10^{-5}\end{array}$ & 1.222075500 & $\begin{array}{c}1.43 \times \\
10^{-8}\end{array}$ \\
\hline 0.6 & 1.560239000 & $\begin{array}{c}7.37 \times \\
10^{-5}\end{array}$ & 1.565813600 & $\begin{array}{c}4.22 \times \\
10^{-8}\end{array}$ & 1.386626200 & $\begin{array}{c}1.63 \times \\
10^{-5}\end{array}$ & 1.393695300 & $\begin{array}{c}1.28 \times \\
10^{-8}\end{array}$ \\
\hline 0.7 & 1.638015900 & $\begin{array}{c}5.36 \times \\
10^{-5}\end{array}$ & 1.642429100 & $\begin{array}{c}1.82 \times \\
10^{-8}\end{array}$ & 1.529416700 & $\begin{array}{c}1.24 \times \\
10^{-5}\end{array}$ & 1.535196300 & $\begin{array}{c}4.97 \times \\
10^{-9}\end{array}$ \\
\hline 1.0 & 1.793582400 & $\begin{array}{c}1.86 \times \\
10^{-6}\end{array}$ & 1.796084600 & $\begin{array}{c}1.25 \times \\
10^{-7}\end{array}$ & 1.817146700 & $\begin{array}{c}4.63 \times \\
10^{-7}\end{array}$ & 1.820257000 & $\begin{array}{c}4.75 \times \\
10^{-9}\end{array}$ \\
\hline
\end{tabular}
imate solution together with its residual error of (25) for different values of $n$ and $\alpha$. From these numerical results, we can obviously observe that such error decreases when $n$ sufficiently increases.

Table 6. Numerical solution of example 13 together with it's residual error for different values of $\alpha$ and $n$

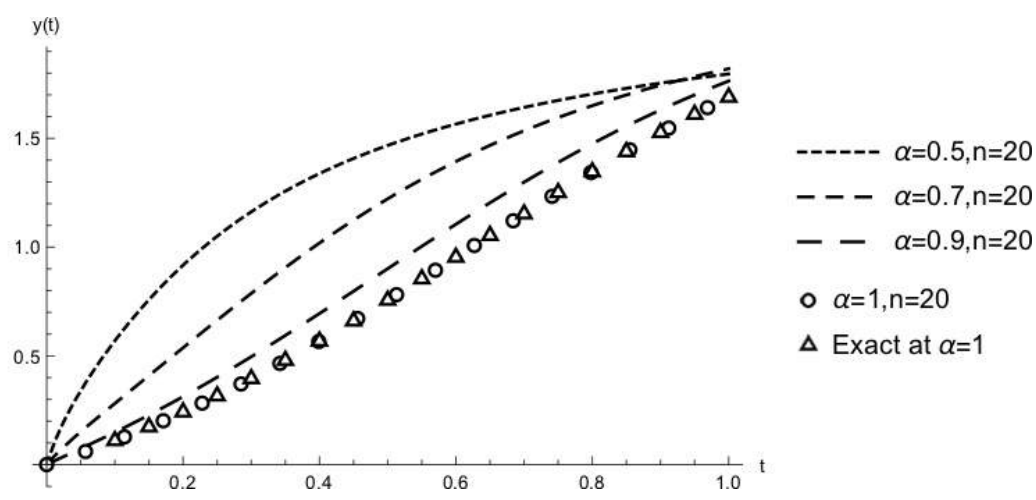

Figure 2. An approximate solution of example 13 for different values of $\alpha$ 
Table 7. Numerical solution of example 14 together with it's residual error for different values of $\alpha$ and $n$

\begin{tabular}{|c|c|c|c|c|c|c|c|c|}
\hline$(\mathrm{r}) 2-3(\mathrm{r}) 4-5(\mathrm{r}) 6-7(\mathrm{r}) 4-5(\mathrm{r}) 8-9$ & \multicolumn{2}{|c|}{$\alpha=1.5, n=10$} & \multicolumn{2}{|c|}{$\alpha=1.5, n=20$} & \multicolumn{2}{|c|}{$\alpha=1.7, n=10$} & \multicolumn{2}{|c|}{$\alpha=1.7, n=20$} \\
\hline 0.1 & 0.009030744 & $\begin{array}{l}1.11 \times \\
10^{-16}\end{array}$ & 0.009030744 & $\begin{array}{l}2.22 \times \\
10^{-16}\end{array}$ & 0.009167872 & $\begin{array}{l}3.33 \times \\
10^{-16}\end{array}$ & 0.009167872 & $\begin{array}{l}1.44 \times \\
10^{-15}\end{array}$ \\
\hline 0.2 & 0.035396410 & $\begin{array}{l}7.44 \times \\
10^{-15}\end{array}$ & 0.035396410 & $\begin{array}{l}2.22 \times \\
10^{-16}\end{array}$ & 0.036019388 & $\begin{array}{l}4.44 \times \\
10^{-16}\end{array}$ & 0.036019388 & $\begin{array}{l}1.55 \times \\
10^{-15}\end{array}$ \\
\hline 0.3 & 0.078049074 & $\begin{array}{l}3.51 \times \\
10^{-13}\end{array}$ & 0.078049074 & $\begin{array}{l}4.44 \times \\
10^{-16}\end{array}$ & 0.079604717 & $\begin{array}{l}1.89 \times \\
10^{-14}\end{array}$ & 0.079604717 & $\begin{array}{l}1.33 \times \\
10^{-15}\end{array}$ \\
\hline 0.5 & 0.208294260 & $\begin{array}{l}4.07 \times \\
10^{-11}\end{array}$ & 0.208294260 & 0 & 0.213363390 & $\begin{array}{l}3.73 \times \\
10^{-12}\end{array}$ & 0.213363390 & $\begin{array}{l}2.00 \times \\
10^{-15}\end{array}$ \\
\hline 0.6 & 0.294056080 & $\begin{array}{l}2.17 \times \\
10^{-10}\end{array}$ & 0.294056080 & $\begin{array}{l}6.66 \times \\
10^{-16}\end{array}$ & 0.301821170 & $\begin{array}{l}2.48 \times \\
10^{-11}\end{array}$ & 0.301821170 & $\begin{array}{l}1.11 \times \\
10^{-15}\end{array}$ \\
\hline 0.7 & 0.392439810 & $\begin{array}{l}8.79 \times \\
10^{-10}\end{array}$ & 0.392439810 & 0 & 0.403580350 & $\begin{array}{l}1.24 \times \\
10^{-10}\end{array}$ & 0.403580350 & $\begin{array}{l}1.33 \times \\
10^{-15}\end{array}$ \\
\hline 1.0 & 0.755609580 & $\begin{array}{c}2.01 \times \\
10^{-8}\end{array}$ & 0.755609580 & $\begin{array}{l}8.88 \times \\
10^{-16}\end{array}$ & 0.781140820 & $\begin{array}{c}5.27 \times \\
10^{-9}\end{array}$ & 0.781140820 & $\begin{array}{l}1.33 \times \\
10^{-15}\end{array}$ \\
\hline
\end{tabular}

\section{CONCLUSION}

In this paper, two efficient power series formulas together with their error terms have been simply derived for the purpose of approximating the Riemann-Liouville fractional derivative operator. It has been shown through addressing several numerical examples that these formulas, which successfully have generated effective series solutions, can be employed to solve many linear and nonlinear problems in the field of fractional calculus.

\section{REFERENCES}

[1] A. Atangana, and J. F. Gómez-Aguilar, " Numerical approximation of Riemann-Liouville definition of fractional derivative: From Riemann-Liouville to Atangana-Baleanu," Numerical Methods for Partial Differential Equations, vol. 34, no. 5, pp. 1-22, 2017, doi: 10.1002/num.22195.

[2] I. M. Batiha, R. B. Albadarneh, S., Momani, and I. H. Jebril, "Dynamics analysis of fractional-order Hopfield neural networks," International Journal of Biomathematics, vol.13, no. 08, pp. 2050083, 2020.

[3] I. M. Batiha, R. El-Khazali, A. AlSaedi and S. Momani, "The General Solution of Singular Fractional-Order Linear Time-Invariant Continuous Systems with Regular Pencils," Entropy, vol. 20, no. 6, 2018, doi: 10.3390/e20060400.

[4] M. D. Patil, K. Vadirajacharya, and K. Swapnil, "Design of fractional order controllers using constrained optimization and reference tracking method," International Journal of Power Electronics and Drive Systems, vol. 11, no. 1, pp. 291, 2020, doi: 10.11591/ijpeds.v11.i1.pp291-301.

[5] T. Amieur, M. Sedraoui, and O. Amieur, "Design of Robust Fractional-Order PID Controller for DC Motor Using the Adjustable Performance Weights in the Weighted-Mixed Sensitivity Problem," IAES International Journal of Robotics and Automation, vol. 7, no. 2, pp. 108-118, 2018, doi: 10.11591/ijra.v7i2.pp108-118.

[6] B. M. Duarte, and J. A. Tenreiro Machado, "Chaotic phenomena and fractional-order dynamics in the trajectory control of redundant manipulators," Nonlinear Dynamics, vol. 29, no. 1, pp. 315-342, 2002, doi: 10.1023/A:1016559314798.

[7] K. B. Oldham, "Fractional differential equations in electrochemistry," Advances in Engineering Software, vol. 41, no. 1, pp. 9-12, 2010, doi: 10.1016/j.advengsoft.2008.12.012.

[8] N. Engheta, "On fractional calculus and fractional multipoles in electromagnetism," IEEE Transactions on Antennas and Propagation, vol. 44, no. 4, pp. 554-566, 1996, doi: 10.1109/8.489308.

[9] R. L. Magin, "Fractional calculus models of complex dynamics in biological tissues," Computers and Mathematics with Applications, vol. 59, no. 5, pp.1586-1593, 2010, doi: 10.1016/j.camwa.2009.08.039.

[10] V. Gafiychuk, B. Datsko and V. Meleshko, "Mathematical modeling of time fractional reaction-diffusion systems," Journal of Computational and Applied Mathematics, vol. 220, no. 1, pp. 215-225, 2008, doi: 10.1016/j.cam.2007.08.011.

[11] A. Atangana and A. Secer, "A note on fractional order derivatives and table of fractional derivatives of some special 
functions," Abstr. Appl Anal., vol. 2013, pp. 1-16, 2013, doi: 10.1155/2013/279681.

[12] M. Caputo, "The memory damped seismograph," Boll. Geofisica Teorica ed Appl., vol. 54, pp. 217-228, 2013, doi: 10.4430/bgta0091.

[13] B. Duan, Z. Zheng and W. Cao, "Spectral approximation methods and error estimates for Caputo fractional derivative with applications to initial-value problems," J. Comput. Phys., vol. 319, pp. 108-128, 2016, doi: 10.1016/j.jcp.2016.05.017.

[14] A. El-Ajou, O. A. Arqub, Z. Al Zhour and S. Momani, "New Results on Fractional Power Series: Theories and Applications," Entropy, vol. 15, no. 12, pp. 5305-5323, 2013, doi: 10.3390/e15125305.

[15] B. Riemann, "The Collected Works of Bernhard Riemann," Dover Publications Inc., New York, United States, 2013.

[16] G. Hardy, "Riemann's form of Taylor's series," Journal of the London Mathematical Society, vol. 1, pp. 48-57, 1945.

[17] J. J. Trujillo, M. Rivero and B. Bonilla, "On a Riemann-Liouville generalized Taylor's formula," Journal of Mathematical Analysis and Applications, vol 231, pp. 255-265 , 1999, doi: 10.1006/jmaa.1998.6224.

[18] Z. M. Odibat and N. T. Shawagfeh, "Generalized Taylor's formula," Applied Mathematics and Computation, vol. 186, no. 1, pp. 286-293, 2007, doi: 10.1016/j.amc.2006.07.102.

[19] K. Oldham and J. Spanier, "The Fractional Calculus Theory and Applications of Differentiation and Integration to Arbitrary Order," Academic Press, San Diego, USA, 1974.

[20] Y. Wei, Y. Chen, Q. Gao and Y. Wang, "Infinite series representation of fractional calculus: theory and applications," 2019 Chinese Automation Congress (CAC), Hangzhou, China, pp. 1697-1702, 2019, doi: 10.1109/CAC48633.2019.8997499.

[21] S. G. Samko, A. A. Kilbas and O. I. Marichev, "Fractional integrals and derivatives: theory and applications," Gordon and Breach Science Publishers, Switzerland, Philadelphia, Pa., USA, 1993.

[22] J. Munkhammar, "Fractional calculus and the Taylor-Riemann series," Rose-Hulman Undergraduate Mathematics Journal, vol 6, no. 1, pp. 2005.

[23] A. Fernandez and D. Baleanu, "The mean value theorem and Taylor's theorem for fractional derivatives with Mittag-Leffler kernel," Advances in Difference Equations, vol. 2018, no. 86, 2018, doi: 10.1186/s13662-018-1543-9.

[24] R. B. Albadarneh, I. M. Batiha and M. Zurigat, "Numerical solutions for linear fractional differential equations of order $1<\alpha<2$ using finite difference method (FFDM)," Journal of Mathematics and Computer Science, vol. 16, no. 1, pp. 103-111, 2016.

[25] R. B. Albadarneh, M. Zurigat and I. M. Batiha, "Numerical solutions for linear and non-linear fractional differential equations," International Journal of Pure and Applied Mathematics, vol. 106, no. 3, pp. 859-871, 2016.

[26] E. Sousa and C. Li, "A weighted finite difference method for the fractional diffusion equation based on the Riemann-Liouville derivative," Appl. Numer. Math., vol. 90, pp. 22-37, 2015, doi: 10.1016/j.apnum.2014.11.007.

[27] T. Bakkyaraj and R. Sahadevan, "Invariant analysis of nonlinear fractional ordinary differential equations with Riemann-Liouville fractional derivative," Nonlinear Dyn., vol. 80, pp. 447-455, 2015, doi: 10.1007/s11071-014-1881-4.

[28] J. S. Leszczyński, "An introduction to fractional mechanics," Publishing Office of Częstochowa University of Technology, Czȩstochowa, Poland, 2011.

[29] A. K. Alomari, G. A. Drabseh, M. F. Al-Jamal, and R. B. Albadarneh, "Numerical simulation for fractional phi-4 equation using homotopy Sumudu approach," International Journal of Simulation and Process Modelling, vol. 16, no. 1, pp. 26-33, 2021, doi: 10.1504/IJSPM.2021.113072.

[30] R. B. Albadarneh, I. M. Batiha, N. Tahat and A. K. Alomari, "Analytical Solutions of Linear and Non-linear Incommensurate Fractional-order Coupled Systems," Indonesian Journal of Electrical Engineering and Computer Science, vol. 21, no. 2, pp. 776-790, 2021, doi: 10.11591/ijeecs.v21.i2.pp776-790.

[31] H. Latifizadeh, "A coupling method of Homotopy technique and Laplace transform for nonlinear fractional differential equations," International Journal of Advances in Applied Sciences, vol. 1, no. 4, pp. 159-170, 2012, doi: 10.11591/ijaas.v1.i4.pp159-170.

[32] K. Diethelm, "The Analysis of Fractional Differential Equations: An Application-Oriented Exposition Using Differential Operators of Caputo Type,” Springer-Verlag Berlin Heidelberg, Braunschweig, Germany, 2004. 


\section{BIOGRAPHIES OF AUTHORS}

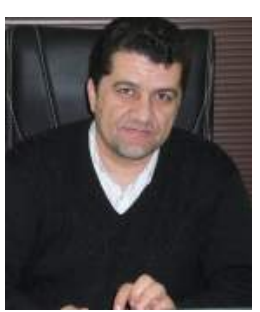

Ramzi B. Albadarneh received his BSc in Mathematics from Al al-Bayt University, Jordan in 2000, and MSc in Pure Mathematics from Al al-Bayt University, Jordan, in 2003. He received a PhD in Mathematics from University of Jordan in 2009. He is an Associate Professor at Department of Mathematics, The Hashemite University. His main research interests in Numerical methods for differential equations, fractional calculus. He has published more than 15 papers, authored/coauthored, in more than 15 refereed journal and conference papers.

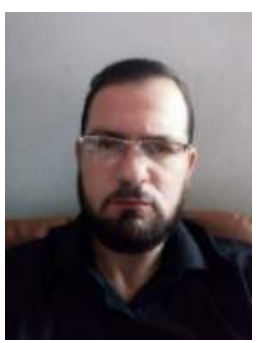

Iqbal Bateha holds a MSc. (2014) from Al al-Bayt University, and a PhD (2018) in applied mathematics from The University of Jordan. He is a founding member of the international center for scientific researches and studies (ICSRS-Jordan), and he is also currently working as an assistant professor at the Department of Mathematics in National Irbid University. He has published several papers in different peer reviewed international journals. Iqbal M. Batiha has been awarded a Grünwald-Letnikov Award: Best Student Papers (theory, application), which was presented from the International Conference on Fractional Differentiation and its Applications (ICFDA'18) that held in Amman, July, 2018. He is currently serving on the board of the International Journal of Open Problems in Computer Science and Mathematics (IJOPCM), and the editorial review boards of two international journals; the International Journal of Systems Science and Applied Mathematics (IJSSAM), and Applied Mathematics and Information Science (AMIS).

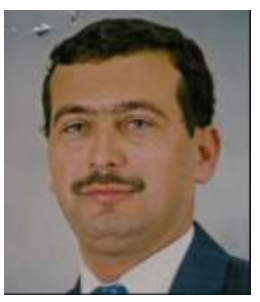

Ahmad Adawi He got B.Sc degree in Mathematics from the University of Jordan in 1987, M.Sc degree in Mathematics from the University of Jordan in 1989, and Ph.D degree in Mathematics from the University of Jordan in 1999. He is an associate professor at the department of Mathematics in the Hashemite University, Jordan. His research interests are differential equations, integral equations and integral transforms.

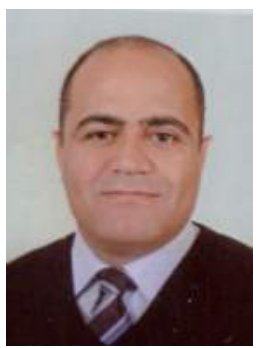

Nedal Tahat He is a Professor in the Department of Mathematics at The Hashemite University - Jordan. Dr. Tahat earned his B.Sc. in Mathematics from Yarmouk University, Jordan in 1994, and M.Sc. in Pure Mathematics from Al al-Bayt University, Jordan, in 1998. He received a PhD in Applied Number Theory (Cryptography) from National University of Malaysia (UKM) in 2010. Prof. Tahat joined The Hashemite University in 2011 and served as the Head of the department of Mathematics. from 2013 to 2016 and also vice - Dean of the faculty of science from 2019until now. His main research interests are cryptology, number theory and applied Mathematics. He has published more than 50 papers, authored/coauthored, and more than 20 refereed journal and conference papers.

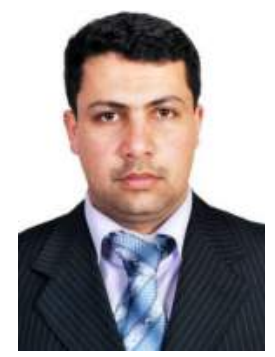

A.K. Alomari received his PhD in Mathematics from the University Kebangsaan Malaysia in 2009, Malaysia. He is an Associate Professor at the Department of Mathematics, Yarmouk University, Irbid, Jordan.His research interests include numerical methods for differential equations. He is an author of a great deal of research studies in at international journals as well as conference proceedings. 